\title{
Fases sodalita formadas a partir de caulinitas amazônicas sob condições de digestão Bayer
}

\author{
Sodalite phases formed from kaolinite \\ under bayer-type conditions
}

\footnotetext{
${ }^{1}$ Programa de Pós-graduação em Geologia e Geoquímica, Laboratório de Caracterização Mineral PPGG-LCM-UFPA CEP: 66075-110, Belém, PA e-mail:eng.caiomelo@hotmail.com

${ }^{2}$ Faculdade de Engenharia de Materiais - UFPA - Campus Ananideua e-mail: paz@ufpa.br; rsangelica@gmail.com
}

\begin{abstract}
RESUMO
As bauxitas metalúrgicas da região de Paragominas-PA (norte do Brasil) comumente apresentam uma alta quantidade de caulinita. Durante a lixiviação da bauxita nas condições de processo Bayer, este mineral reage com $\mathrm{NaOH}$ formando indesejavelmente sodalita, o qual é insolúvel e, portanto, descartada com o resíduo sólido, causando significativa perda do agente lixiviante. Neste trabalho foi dada a atenção a dois tipos principais de sodalita formadas em função de diferenças na concentração caustica e tempo de reação. Assim, o processo hidrotérmico foi conduzido em temperatura de $150{ }^{\circ} \mathrm{C}$, concentração caustica variando de 2,5 à 5,0 M e tempo variando de 60 à 420 min. Os resultados mostraram que estas variáveis afetam tanto a velocidade da transformação da caulinita em sodalita como a cristaloquímica desta fase, resultando na formação de dois tipos de sodalita: 1) sodalita básica tetrahidratada, fase mais estável formada inicialmente e remanescente em todas as condições do processo; e 2) hidrosodalita, a qual é a fase secundária, menos estável e observada apenas nas condições intermediárias. Esta última fase é mais desejável no contexto Bayer devido a menor quantidade de $\mathrm{NaOH}$ consumida. Observou-se que o favorecimento da formação desta fase depende de maiores tempos, concentração caustica e quantidade de caulinita.
\end{abstract}

Palavras-chave: sodalita básica; hidrosodalita; caulim; bauxita.

\begin{abstract}
Metallurgical bauxites from Paragominas-PA region (northern Brazil) commonly exhibit high amounts of kaolinite. During caustic leaching of bauxite in the Bayer process conditions, this mineral reacts with $\mathrm{NaOH}$ and undesirable sodalite is formed, which is insoluble and therefore discarded with the solid residue, causing significant losses of the leaching agent. In the present work, an attention was given to two main types of sodalite formed as function of differences in caustic concentration and residence time. Thus, the hydrothermal process was carried out at temperature of $150{ }^{\circ} \mathrm{C}$, caustic concentration ranging from 2.5 to $5.0 \mathrm{M}$ and time varying from 60 to $420 \mathrm{~min}$. Results showed that these variables affect both the speed of kaolinite to sodalite transformation as the crystal chemistry of the two main types of sodalite phases formed: 1) tetrahydrate basic sodalite, which is the more stable phase initially formed and that remains during all process conditions; and 2) hydrosodalite, which is the secondary phase, less stable and observed only in intermediate conditions. This last phase is more desirable in the Bayer context due the less amount of $\mathrm{NaOH}$ consumed. The favoring of this phase formation depends on the long time, caustic concentration and the amount of kaolinite.
\end{abstract}

Keywords: basic sodalite; hydrosodalite; kaolin; bauxite.

\section{INTRODUÇÃO}

Em um contexto nacional o Estado do Pará destaca-se pelo seu potencial mineral, representando uma liderança ( 90 \%) na produção de caulim para cobertura de papel e bauxita metalúrgica. No cenário mundial, o Pará faz o Brasil se destacar entre os maiores produtores mundiais dos dois tipos de bens minerais mencionados. 
Dessa maneira, a atividade mineradora dessas commodities tem um importante papel na economia do estado, assim como, na economia do país e do mundo [1]. Ambos têm origem supergênica, isto é: são produtos do processo de hidrólise de rochas aluminossilicatadas, sendo a gênese destes relacionada ao mesmo ambiente, condições climáticas, relevo e vegetação [2-6].

O mineral útil do caulim é a caulinita e das bauxitas paraenses a gibbsita. Os minerais de ganga comuns nos caulins amazônicos são: quartzo, anatásio, mica e hematita; nas bauxitas: caulinita, hematita, Algoethita, anatásio e quartzo. Assim, para atender as especificações de produto, os dois minérios são submetidos a processos de beneficiamento em indústrias instaladas no Pará, e inevitavelmente, oneram grandes áreas, chamadas de lagoas de sedimentação ou barragens, para deposição do rejeito mineral de característica fina (pequeno tamanho de partícula). Característica essa que dificulta a recuperação vegetal da área [3-9].

No caso do caulim, o beneficiamento é realizado para separar o quartzo (etapa de desareiamento), os minerais que afetam a alvura (etapa de clarificação) e a caulinita de tamanho de partícula inadequada para cobertura de papel (etapa de centrifugação) [8, 9].

No caso da bauxita, o beneficiamento é realizado para separar a caulinita (pelo processo de desagregação/lavagem). A caulinita é o mineral deletério no processo metalúrgico para purificação da gibbsita (processo Bayer), e nesse contexto é conhecida como sílica reativa, pois durante esta etapa é rapidamente lixiviada e posteriormente precipitada na forma de sodalita [6]. Esta reação é irreversível e indesejável, pois grande quantidade de $\mathrm{NaOH}$ (agente lixiviante do processo) é descartada na forma de sodalita na lama vermelha. Estima-se que o processamento de bauxitas com teores de caulinita $>5 \%$ aumentem cerca de $20 \%$ os custos do processo [6, 10, 11].

As sodalitas sintéticas são fases zeolíticas que apresentam como fórmula geral: $\mathrm{M}_{\mathrm{m}}\left[\mathrm{TO}_{2}\right]_{\mathrm{t}} \mathrm{X}_{\mathrm{x}} \cdot n \mathrm{H}_{2} \mathrm{O}$, onde T representa os átomos da unidade principal, geralmente (Al, Si, Ge, Ga e B); X são espécies aniônicas que ocupam a unidade secundária $\left(\mathrm{OH}^{-}, \mathrm{Cl}^{-}, \mathrm{Br}^{-}, \mathrm{CO}_{3}{ }^{2-}, \mathrm{SO}_{4}{ }^{2-}\right.$, etc.); $\mathrm{M}$ são espécies catiônicas compensadores da carga da estrutura, que em geral são átomos alcalinos ou alcalinos terrosos (mais comumente $\mathrm{Na}^{+}, \mathrm{K}^{+}$, $\mathrm{Ca}^{2+}$ e $\mathrm{Mg}^{2+}$ ); $n$ representa a hidratação, sendo $0 \leq \mathrm{n} \leq 8$ [10,11]. Apresenta uma estrutura tridimensional cúbica do grupo espacial $\mathrm{P} \overline{4} 3 \mathrm{n}$, com parâmetro de cela unitária $a_{0} \sim 9,0 \AA$, formada pela coordenação tetraédrica de átomos $\mathrm{T}$ com oxigênios ligados a outros átomos $\mathrm{T}$ (T-O-T), originando uma conexão de cantos compartilhados $\mathrm{TO}_{4}$ tetraédricos. A sodalita é estruturada pela formação e sequenciamento de anéis simples de 6 tetraedros, formando uma cavidade octaédrica tridimensional. O truncamento ordenado e regular dessas cavidades (chamadas de $\beta$-cavidade, também conhecidas como cavidade-sodalita) pode ocorrer de diferentes maneiras, assim formando estruturas zeolíticas em comum: Sodalita (SOD), zeólita A (LTA), zeólitas X/Y (FAU) e EMT [9].

Com o objetivo de produzir zeólitas para diversas finalidades e ao mesmo tempo agregar valor à materiais hoje sem aproveitamento - rejeitos do beneficiamento do caulim e bauxita - vários trabalhos vêm sendo realizados utilizando como matéria-prima tais rejeitos como fonte de $\mathrm{Al}_{2} \mathrm{O}_{3}$ e $\mathrm{SiO}_{2}$ (na forma de caulinita) para as sínteses [3, 7-9, 12]. Embora estes estudos tenham se mostrados promissores em suas propostas, existem poucos trabalhos que foquem nos fatores físico-químicos que afetam a formação de sodalita, bem como características químicas e estruturais dessa fase formada a partir das caulinitas presentes nos caulins e bauxitas da região [13-15]. Assim, há certa carência de dados que deem suporte a estudos que visem reduzir o consumo de sódio do processo Bayer, bem como, viabilizar o processamento das bauxitas com alta sílica reativa.

Dessa forma, este trabalho tem como objetivo investigar a relação das variáveis: concentração cáustica e tempo de reação com as sodalitas formadas a partir de caulinitas da região de Paragominas-PA sob condições de digestão tipo-Bayer. Assim, gerar informações dessa fase “vilã” na produção de alumina metalúrgica, que podem ser úteis a nortear trabalhos futuros que objetivem a diminuição dos custos do processo Bayer e a viabilização econômica das bauxitas com alta sílica reativa da região amazônica.

\section{MATERIAIS E MÉTODOS}

\subsection{Material de Partida}

Dois materiais cauliníticos foram utilizados nesse trabalho: um caulim e uma ganga do beneficiamento da bauxita.

O caulim, beneficiado pela empresa Imerys Rio Capim Caulim (IRCC S/A), a qual explora caulim da região do Capim, município de Ipixuna-PA. Este caulim apresenta alta pureza e predominância de partículas com granulometria $<2 \mu \mathrm{m}$, e representa o produto da empresa para cobertura de papel, campanha 2011.

A ganga caulinítica, obtida da lavagem de $12 \mathrm{~kg}$ de bauxita proveniente de um poço de pesquisa reali- 
zado na mina Miltônia 3, Paragominas-PA. A lavagem foi realizada em equipamento Hydro-Clean (Marca Haver \& Boecker, modelo de laboratório) seguida de classificação a $0,037 \mathrm{~mm}$ em peneirador vibratório (Marca Abronzinox, com peneiras quadradas de 50 x $50 \mathrm{~cm}$ ) e deslamagem da fração passante em 0,037 mm em hidrociclone Krebbs de 25 mm de diâmetro operando a 25 psi com corte de $10 \mu \mathrm{m}$. A fração overflow dessa etapa corresponde a ganga caulinítica desse trabalho.

\subsection{Processo hidrotermal}

Todos os ensaios foram realizados em autoclaves de aço inoxidável revestidas internamente de teflon com capacidade máxima de $50 \mathrm{~mL}$ e pressão autógena, em temperatura de $150{ }^{\circ} \mathrm{C}$. O estudo foi conduzido utilizando $1 \mathrm{~g}$ do material de partida e um volume de $25 \mathrm{~mL}$ de solução de $\mathrm{NaOH}$ (marca VETEC). As concentrações da solução cáustica foram: 2,5; 3,0; 3,5; 4,0; 4,5 e 5,0 M. Essas condições foram reproduzidas nos tempos de 60, 120, 180 e 420 minutos.

Após o processo hidrotermal, os produtos sólidos foram lavados até $\mathrm{pH} \sim 7$, filtrados e secos em estufa à $105^{\circ} \mathrm{C}$ por $6 h$.

\subsection{Caracterização dos materiais}

Os materiais de partida foram caracterizados por difratometria de raios X (DRX) e por espectroscopia de emissão atômica com plasma (ICP-OES). Os produtos sólidos foram analisados por DRX, ICP-OES, análise termodiferencial e termogravimétrica (ATD/TG) e espectroscopia de absorção na região do infravermelho (EIV).

As análises de DRX foram realizadas em difratômetro modelo X'PERT PRO MPD da PANalytical, tubos de raios X cerâmico e anodos de $\mathrm{Cu}(\mathrm{K} \alpha 1=1,540598 \AA$ \&̊), com foco fino longo (2200 W- $60 \mathrm{kV})$, filtro $\mathrm{K} \beta$ de níquel. As condições instrumentais utilizadas foram: varredura de $5^{\circ}$ a $100^{\circ} \mathrm{em} 2 \theta$; voltagem de $40 \mathrm{kV}$ e corrente de $40 \mathrm{~mA}$; tamanho do passo: $0,02^{\circ}$ em $2 \theta$ e 90 s o tempo/passo; fenda divergente de $1 / 4^{\circ}$ e antiespalhamento de $1 / 2^{\circ}$; máscara de $10 \mathrm{~mm}$.

O software X'Pert High Score versão 3.0, também da PANalytical, foi usado na identificação de fases.

As análises químicas (elementos maiores) foram realizadas por ICP-OES em laboratório comercial ACME LabsTM com a fusão de $0,2 \mathrm{~g}$ de amostra com borato de lítio, seguida de digestão ácida. A perda ao fogo foi realizada por calcinação das amostras à $1000^{\circ} \mathrm{C}$ após secagem das amostras em $105^{\circ} \mathrm{C}$.

As análises de ATD/TG foram realizadas em um equipamento modelo PL Thermal Science com analisador térmico simultâneo STA1000/1500, da Stanton Redcroft Ltda, com forno cilíndrico vertical, conversor digital acoplado a um microcomputador, utilizando uma faixa de temperatura entre 25 e $1100{ }^{\circ} \mathrm{C}$, com taxa de aquecimento de $10{ }^{\circ} \mathrm{C} \cdot \mathrm{min}^{-1}$ em atmosfera estática do ar.

As análises de EIV foram realizadas utilizando um Espectrômetro Thermo modelo IR100. A região espectral analisada foi de 4000-400 $\mathrm{cm}^{-1}$, média de 64 medidas com resolução de $4 \mathrm{~cm}^{-1}$. As amostras foram preparadas no modo de pastilha na proporção de $1 \mathrm{mg}$ de amostra para $150 \mathrm{mg}$ de $\mathrm{KBr}$.

\section{RESULTADOS E DISCUSSÃO}

\subsection{Caracterização do material de partida}

A Figura 1 apresenta os difratogramas das amostras de caulim e da ganga caulinítica. Observa-se que a amos-

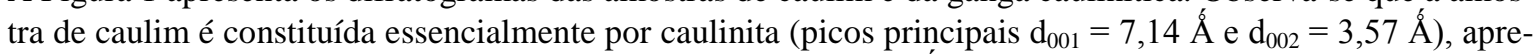
sentando um único pico de anatásio (pico principal $\mathrm{d}_{011}=3,52 \AA$ ) , o qual é um mineral acessório que, em geral, está sempre presente em pequena quantidade nas amostras de caulim da região, característico da sua origem geológica [8]. Observa-se na região ${ }^{\circ} 2 \theta$ entre $19,5^{\circ}$ à $23^{\circ}$ os picos bem definidos da região característica do "triplet" da caulinita, com índice de Hinkley $(\mathrm{IH})=1,75$, confirmando que a amostra possuí bom ordenamento estrutural [16].

No difratograma da amostra da ganga caulinítica as fases identificadas foram: caulinita $\left(\mathrm{d}_{001}=7,14 \AA\right.$

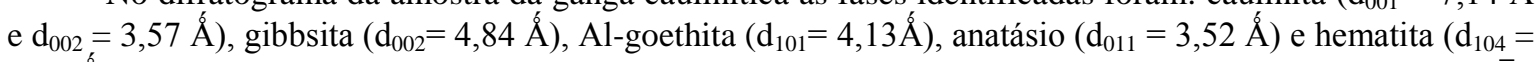
$2,69 \AA$ Á). $\mathrm{O}$ índice de Hinkey para a caulinita presente não foi calculado devido a sobreposição dos picos $1 \overline{1} 0$ e $11 \overline{1}$ (caulinita) com os picos $11 \overline{1}$ e $20 \overline{2}$ da gibbsita e 101 da goethita. No entanto, os valores obtidos de $F W H M$ das reflexões basais $\left(F W H M_{001}=0,146\right.$ e $\left.F W M H_{002}=0,146\right)$ mostraram que a caulinita presente nessa amostra também apresenta bom ordenamento estrutural. 

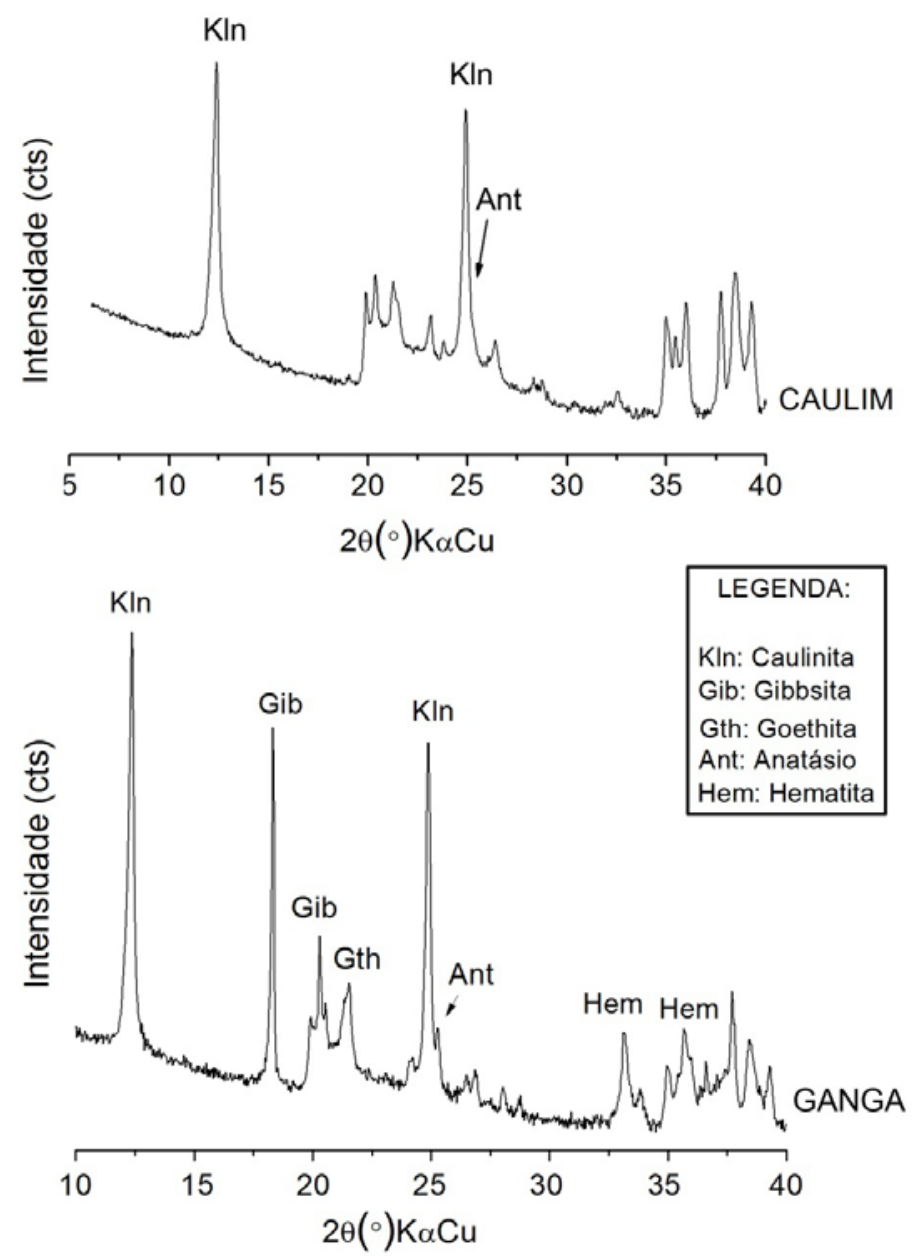

Figura 1: Difratograma dos materiais de partida: caulim e ganga caulinítica

A Tabela 1 apresenta os valores obtidos pela análise química dos materiais de partida. Pode-se observar que na amostra do caulim a $\mathrm{Al}_{2} \mathrm{O}_{3}$ e $\mathrm{SiO}_{2}$ representam 84,35 \% dos componentes da amostra, assim, somados aos 14,14 \% da perda ao fogo, tem-se 98,49 \%, estando assim em boa concordância com a caulinita teórica, confirmando que a mesma é essencialmente constituída de caulinita [8]. Na amostra da ganga todo o valor de $\mathrm{SiO}_{2}$ corresponde à caulinita, e a perda ao fogo corresponde aos minerais: caulinita, goethita e gibbsita.

Tabela 1: Composição química do caulim e da ganga caulinítica.

\begin{tabular}{l|l|l|l}
\hline CONSTITUINTES (\%) & CAULIM & GANGA & CAULINITA TEÓRICA \\
\hline $\mathrm{SiO}_{2}$ & 45,21 & 27,21 & 46,54 \\
\hline $\mathrm{Al}_{2} \mathrm{O}_{3}$ & 39,14 & 37,45 & 39,50 \\
\hline $\mathrm{Fe}_{2} \mathrm{O}_{3}$ & 0,64 & 15,15 & - \\
\hline $\mathrm{TiO}_{2}$ & 0,41 & 2,15 & - \\
\hline Outros & 0,43 & 0,18 & - \\
\hline P.F & 14,14 & 17,7 & 13,96 \\
\hline
\end{tabular}

P.F: perda ao fogo.

\subsection{Produtos sólidos}

A destruição/conversão da caulinita em sodalita em meio fortemente alcalino já é bastante conhecida e estudada [17-20]. A caulinita é dissolvida pela solução de $\mathrm{NaOH}$ formando um licor de aluminato e silicato de sódio que rapidamente se precipita na forma de sodalita (insolúvel nas condições tipo-Bayer). 
A influência do tempo de reação é observada na Figura 2, a qual apresenta uma comparação das amostras produzidas na maior concentração estudada $(5,0 \mathrm{M})$ em diversos tempos. É possível observar que uma conversão completa da caulinita em sodalita só é alcançada em tempos maiores que 120 min. A Figura 3 ilustra uma comparação dos difratogramas dos produtos sólidos nas seis concentrações de $\mathrm{NaOH}$ estudadas no tempo de 120 min. Pode-se observar que a transformação de caulinita (diminuição da intensidade do $\mathrm{d}_{001}$ ) em sodalita (aumento da intensidade do $\mathrm{d}_{011}$ ) é fortemente influenciada pela concentração da solução lixiviante, de modo que há uma visível tendência no aumento da velocidade de conversão quando maiores concentrações alcalinas são empregadas.

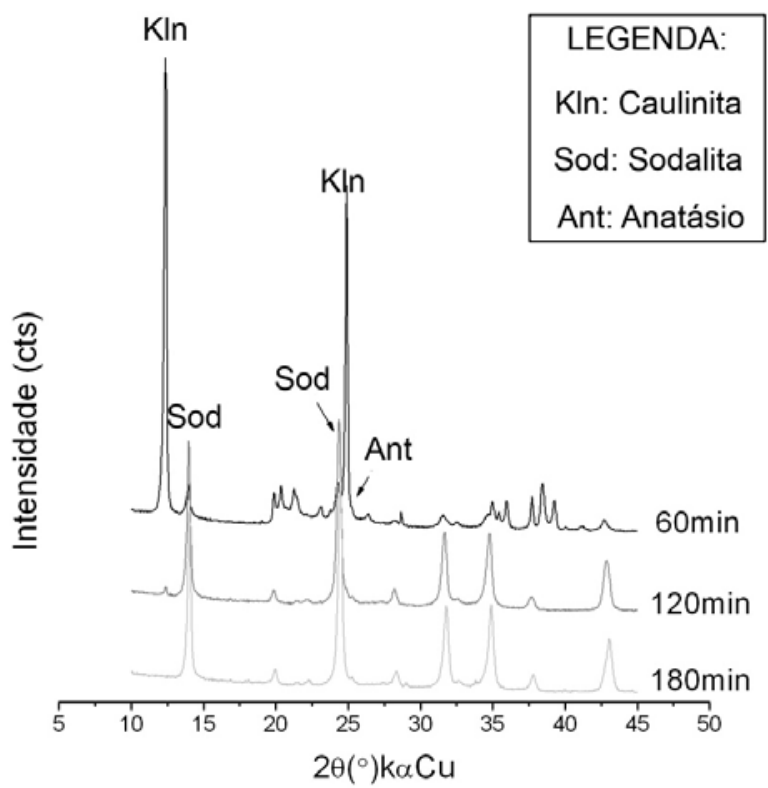

Figura 2: Comparação dos difratogramas dos produtos a partir do caulim em 5,0M: variação do tempo de reação

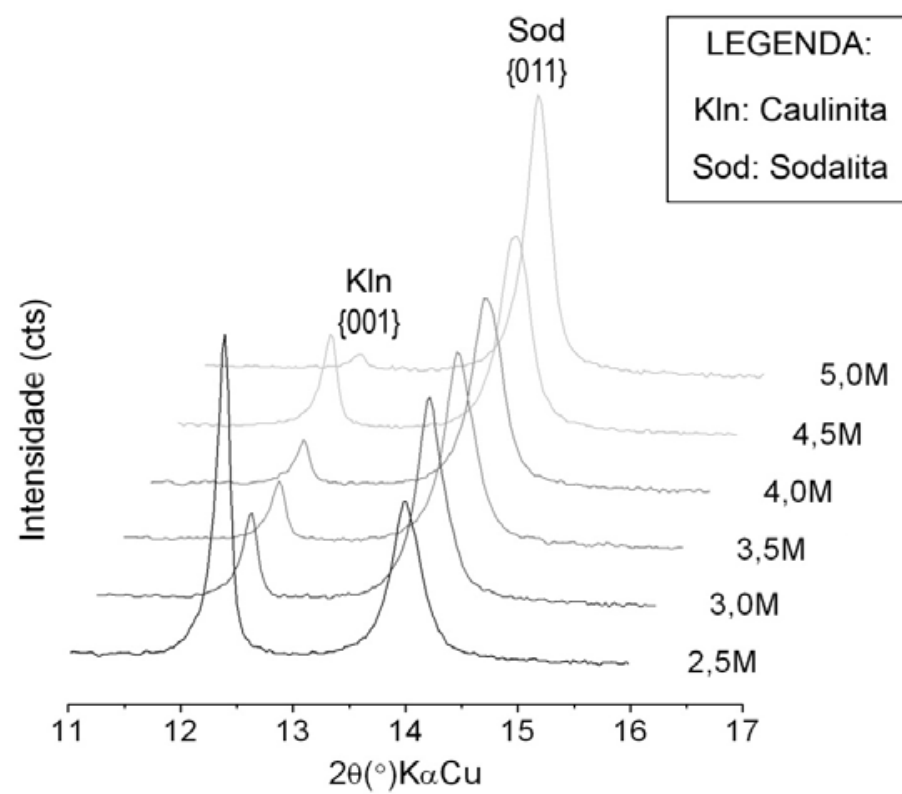

Figura 3: Comparação dos difratogramas dos produtos a partir do caulim em 120 min: variação da concentração molar

A sobreposição dos difratogramas possibilitou a identificação de duas fases sodalita distintas. Os resultados revelaram que a variação da concentração molar da solução de $\mathrm{NaOH}$ e do tempo de reação influenciam na cristaloquímica das fases sodalitas formadas. Tal distinção é identificada pelo deslocamento do padrão difratométrico entre as fases identificadas como $\operatorname{Sod}_{1}$ e $\operatorname{Sod}_{2}$ (Figura 4).

A partir da análise dos difratogramas (Figura 4), das curvas de ATD (Figura 5) e espectros vibracio- 
nais (Figura 6), pode-se dizer que a fase $\operatorname{Sod}_{1}$ é formada nos primeiros momentos da reação, sendo assim, predominante nas amostras de menor concentração e tempo reacional. Esta fase se mantém mais estável e é observada em todas as amostras. A fase $\mathrm{Sod}_{2}$, em contrapartida, é observada como uma fase secundária, menos estável e predominante no tempo de $180 \mathrm{~min}$.

A região do difratograma entre 42,0 e 44,0 $2 \theta$, destacada na Figura 4, apresenta os picos $\mathrm{d}_{033}$ das fases $\operatorname{Sod}_{1}\left(\sim 42,7^{\circ}\right)$ e $\operatorname{Sod}_{2}\left(\sim 43,3^{\circ}\right)$. Essa diferença no valor do $\mathrm{d}_{033}$ resulta de uma variação química com respeito as moléculas de $\mathrm{NaOH}$ e $\mathrm{H}_{2} \mathrm{O}$ que ocupam a porção $\beta$-cavidade da estrutura cristalina da sodalita.

A partir das técnicas de análise adotadas pode-se constatar que as fases identificadas como $\operatorname{Sod}_{1} \mathrm{e}$ $\mathrm{Sod}_{2}$ são, respectivamente, membros extremos de maior hidratação de duas séries de sodalita: básica e não básica.

Da série básica a fase de maior hidratação é a sodalita básica tetrahidratada $\left(\mathrm{Na}_{8}\left[\mathrm{AlSiO}_{4}\right]_{6}\right.$ $\left.[\mathrm{OH}]_{2} \cdot 4 \mathrm{H}_{2} \mathrm{O}\right)$. É reportado [21] que as moléculas que ocupam a cavidade desta fase se apresentam na configuração de um cátion complexo $\left[\mathrm{HNa}_{4}(\mathrm{OH})_{3 \square} \mathrm{H}\right]^{3+}$ onde o centro da estrutura é ocupado por um átomo de hidrogênio, resultando em uma estrutura com dimensão da cela unitária ao 8,93 Å.

Da série não básica a fase de maior hidratação é a hidrosodalita $\left(\mathrm{Na}_{6}\left[\mathrm{AlSiO}_{4}\right]_{6} \cdot 8 \mathrm{H}_{2} \mathrm{O}\right)$. Por não haver $\mathrm{OH}^{-}$esta fase rica em água contém apenas 6 átomos de $\mathrm{Na}$ balanceando a estrutura. Estes constituintes da $\beta$ cavidade se dispõe numa configuração de um cátion complexo di-tetraédrico $\left[\mathrm{Na}_{3}\left(\mathrm{OH}_{2}\right)_{4}\right]^{3+}$. Essa configuração resulta em uma intensa interação (pontes de hidrogênio) entre as moléculas de água e os oxigênios da estrutura, o que por sua vez, resulta em uma contração da dimensão da cela unitária 8,848 $\AA$ [21, 22].

Nas curvas de ATD dos produtos apresentadas na Figura 5 são observados em todas as amostras os eventos térmicos característicos da sodalita básica $\left(\mathrm{Na}_{8}\left[\mathrm{AlSiO}_{4}\right]_{6}[\mathrm{OH}]_{2} \cdot 4 \mathrm{H}_{2} \mathrm{O}\right)$ a qual ocorre em duas etapas. Estima-se que há perda de 1,5 molécula de água em $\sim 150{ }^{\circ} \mathrm{C}$, seguida por uma completa perda de água resultando na formação da fase anidra conhecida como hidroxosodalita $\left(\mathrm{Na}_{8}\left[\mathrm{AlSiO}_{4}\right]_{6} \mathrm{OH}_{2}\right)$ em $\sim 370{ }^{\circ} \mathrm{C}$ [21-23]. Entre 700 e $950{ }^{\circ} \mathrm{C}$ ocorre uma nova perda de massa referente a desidroxilação e transformação estrutural desta fase para carnegieita $\left(\mathrm{NaAlSiO}_{4}\right)$, e em seguida, entre 900 e $1000{ }^{\circ} \mathrm{C}$ observa-se um segundo pico exotérmico referente a transformação polimórfica da carnegieita em nefelina $\left(\mathrm{NaAlSiO}_{4}\right)$ [20-22].

Já na a hidrosodalita $\left(\mathrm{Na}_{6}\left[\mathrm{AlSiO}_{4}\right]_{6} \cdot 8 \mathrm{H}_{2} \mathrm{O}\right)$ entre $100-300{ }^{\circ} \mathrm{C}$ há a liberação das 8 moléculas de água de hidratação resultando em uma perda de massa de aproximadamente $14 \%$ e na formação da forma anidra da sodalita $\left(\mathrm{NaAlSiO}_{4}\right)$. Por não possuir hidroxilas não há perda de massa após $300{ }^{\circ} \mathrm{C}$ como na sodalita básica, apenas a transformação polimórfica em carnegieita em $\sim 900{ }^{\circ} \mathrm{C}[22,24,25]$. 

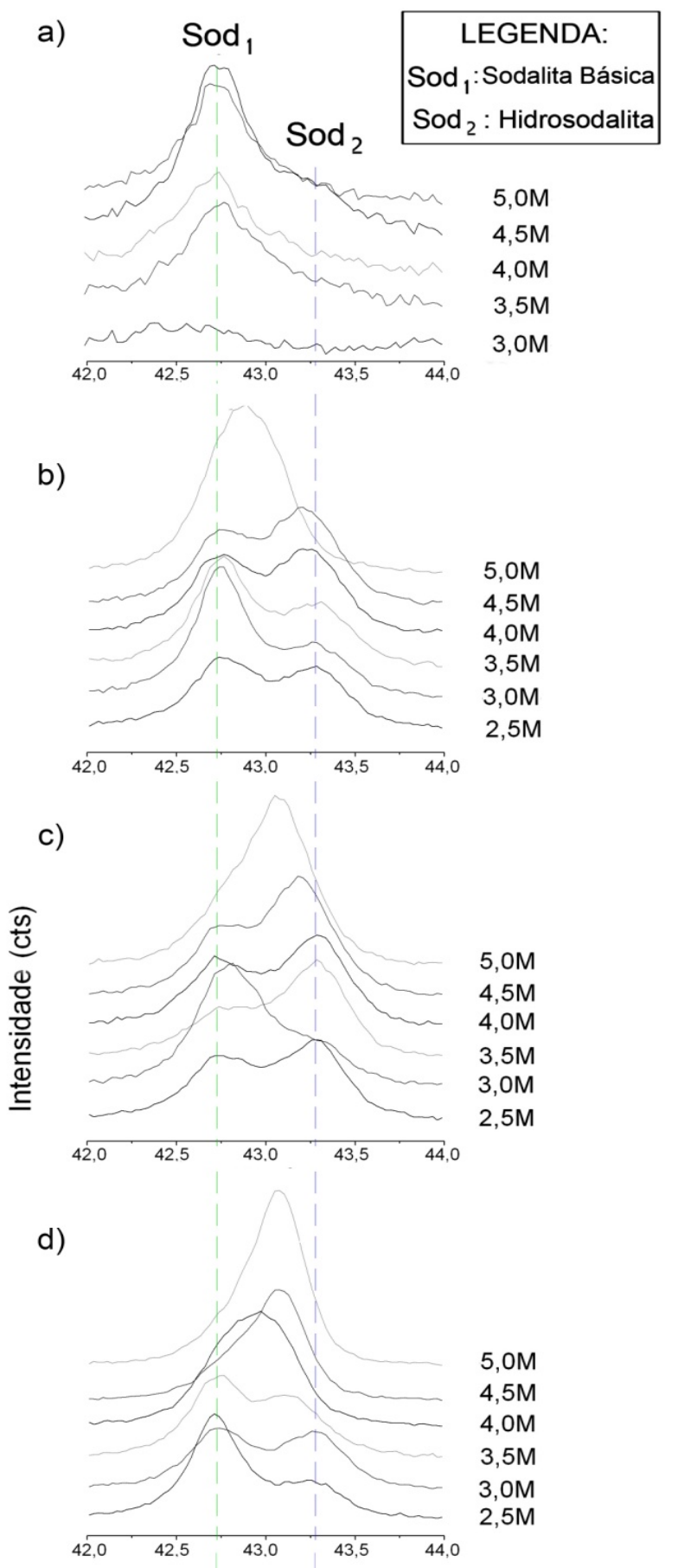

e)

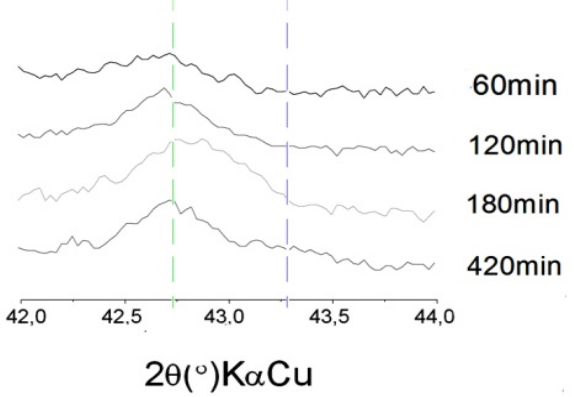

Figura 4: Comparação das sodalitas com a variação da concentração a partir do caulim em a) 60 min; b) 120 min; c) 180 min; d) 420 min; e do tempo a partir da ganga caulinítica em e) 5,0M 

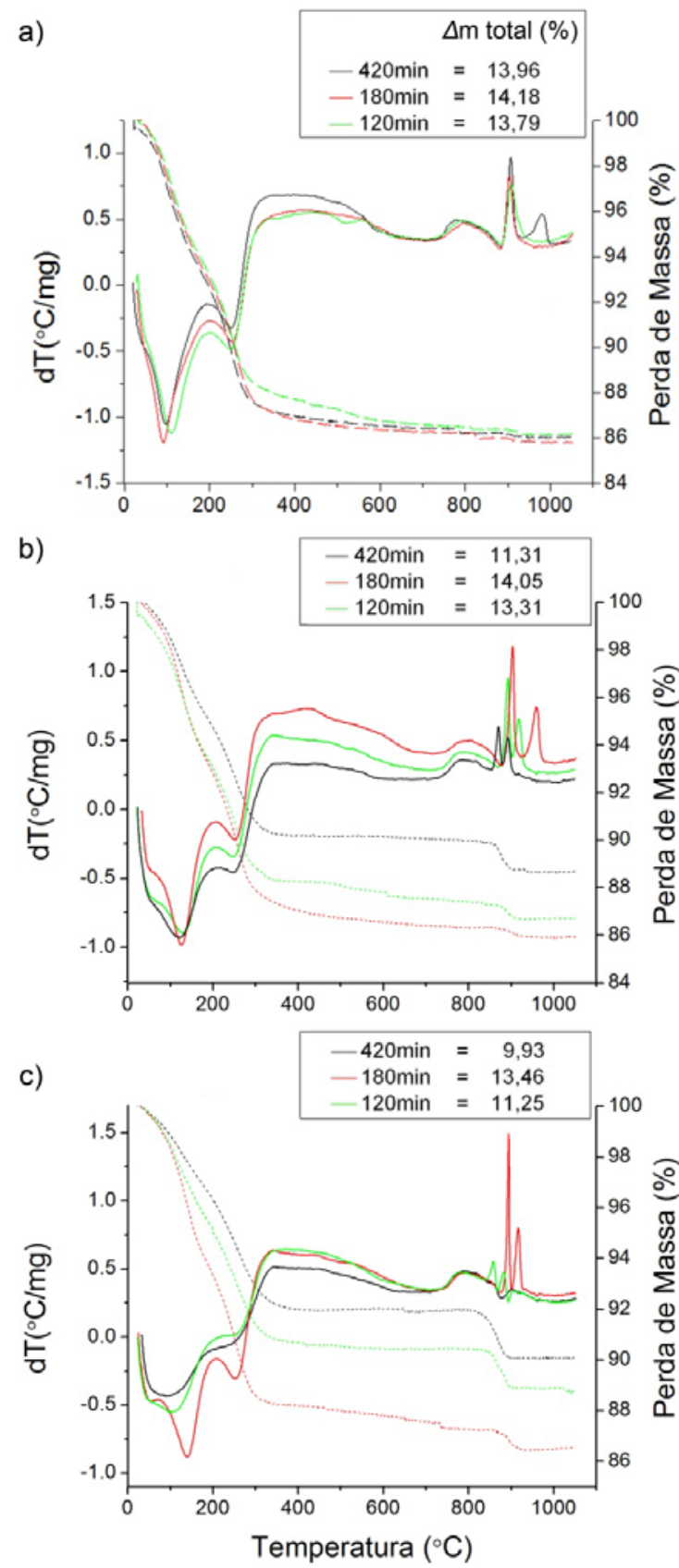

Figura 5: ATD/TG dos produtos a partir do caulim formados em: a) 3,0 M b) 4,0 M e c) 5,0 M. ( $\Delta \mathrm{m}$ total = perda de massa total)

Os espectros vibracionais das fases sodalitas formadas (Figura 6) também mostram bandas de absorção características da sodalita básica (990, 720, 700, 660, 465 e $430 \mathrm{~cm}^{-1}$ ), onde as bandas em 720 e $700 \mathrm{~cm}^{-1}$ correspondem a vibração da ligação Al-O, a banda em $465 \mathrm{~cm}^{-1}$ a vibração da ligação Si-O e as bandas próximas a 1000 e $430 \mathrm{~cm}^{-1}$ correspondem a vibrações das ligações e T-O-T, onde T= Si e Al [26, 27]. A presença do grupo funcional $\mathrm{OH}^{-}$(bandas situadas entre 3500 e $3400 \mathrm{~cm}^{-1}$ ) nestas amostras, revelam a presença de água zeolítica, e caracterizam a presença da sodalita básica. A presença de $\mathrm{H}_{2} \mathrm{O}$ livre é observada pelas bandas nas regiões entre 3400 e $3000 \mathrm{~cm}^{-1}$ e $1650 \mathrm{~cm}^{-1}$ [9, 22].

Como observado nos difratogramas, concentrações alcalinas e tempos intermediários de reação favoreceram a cristalização da hidrosodalita, porém, nenhuma dessas amostras apresentou exclusivamente esta fase, sendo também observado sodalita básica tetrahidratada. Assim os eventos térmicos da desidratação (ATD/TG), bem como as bandas de absorção relacionadas a água (EIV) não fornecem informações de apenas uma fase, dificultando assim a distinção entre elas.

No entanto, pode-se constatar pela maior perda de massa até $400{ }^{\circ} \mathrm{C}$ e a pequena perda após $700{ }^{\circ} \mathrm{C}$ 
(desidroxilação) nas amostras de 180 min que há predominância da hidrosodalita nessas amostras, e da sodalita básica nas demais. De forma semelhante a observação do comportamento das bandas referentes a água livre nas amostras possibilita associar a fase majoritária em cada amostra. Assim, as amostras com maior quantidade de água são as produzidas em 180 min, as quais a fase hidrosodalita é predominante. As amostras de 120 e 420 min apresentaram menores bandas de absorção referentes a água, o que de fato está associado a fase majoritária nessas amostras: sodalita básica tetrahidratada.

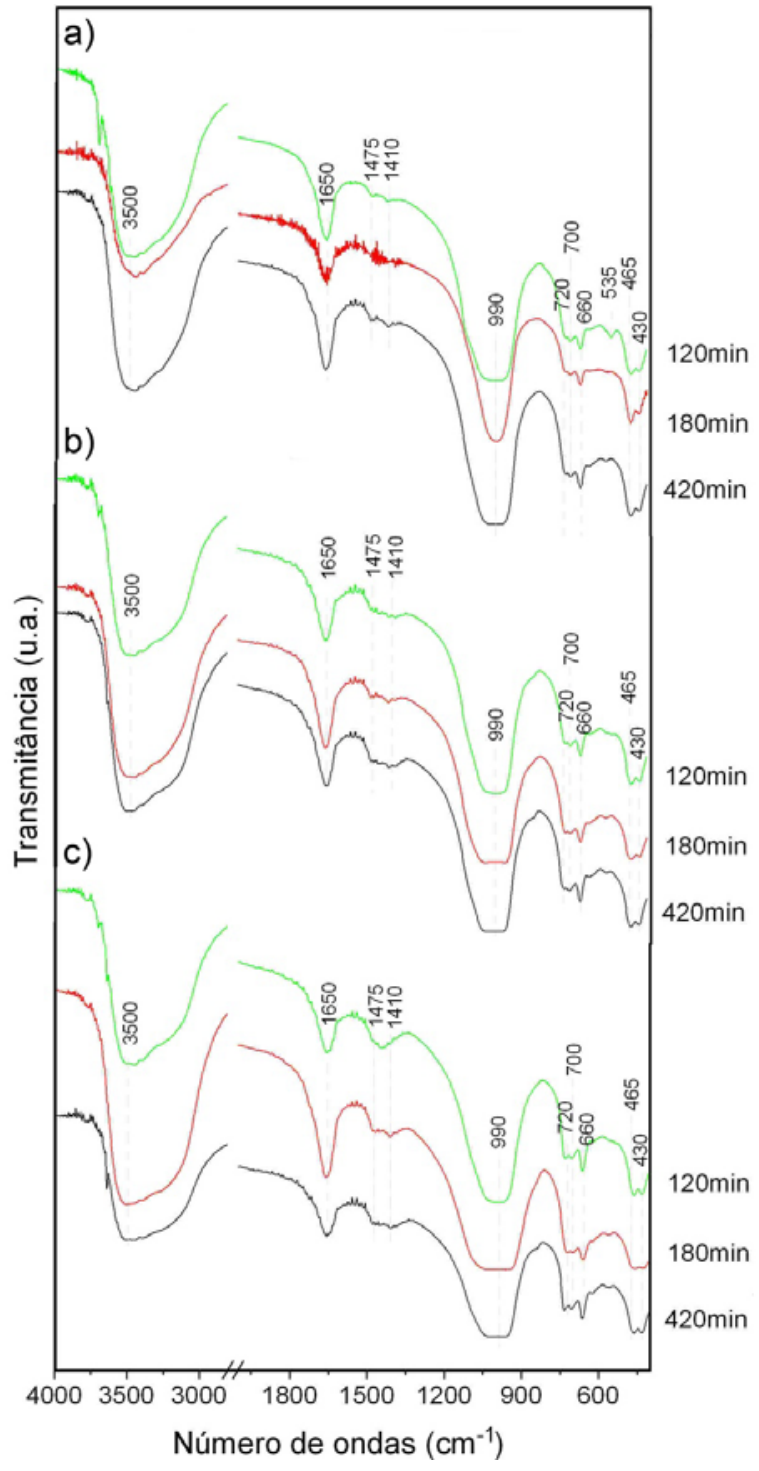

Figura 6: Espectros vibracionais das amostras dos produtos a partir do caulim formados em: a) 3,0 M; b) 4,0 M e; c) 5,0 M

Na Tabela 2 estão sumarizados os resultados da análise química das amostras produzidas em 180 min e os valores estimados teoricamente das sodalitas básicas di e tetrahidratadas e hidrosodalita.

A partir da comparação dos valores medidos de $\mathrm{SiO}_{2}, \mathrm{Al}_{2} \mathrm{O}_{3}, \mathrm{Na}_{2} \mathrm{O}$ e P.F. com os valores teóricos das sodalitas, se observa que a fase predominante nas amostras produzidas em 180 min de fato é a hidrosodalita.

Vale ressaltar que os valores obtidos para a amostra da ganga caulinítica mais se diferenciam dos teóricos. Isto é devido a concentração dos minerais acessórios presentes na bauxita os quais representam $19 \%$ dos elementos identificados na análise química, além da contribuição da perda ao fogo da goethita e/ou amorfos ocasionalmente presentes.

Nota-se ainda que embora a hidrosodalita tenha sido identificada nas amostras de ganga cauliníticas produzidas em 5,0 M e nos tempos de 180 min e 420 min, diferentemente das amostras a partir do caulim, a fase majoritária foi a sodalita básica tetrahidratada. Isto também ocasionado pela presença dos minerais aces- 
sórios da ganga caulinítica derivada da bauxita, de forma que para 1 g de material de partida utilizado, a quantidade de caulinita na ganga é bem inferior a presente no caulim (o qual é essencialmente constituído de caulinita). Assim, constata-se que menores concentrações de caulinita no meio reacional não favorecem a nucleação da hidrosodalita e, portanto, a sodalita básica é predominante.

Tabela 2: Análise química dos produtos formados em 180 min (sodalitas teóricas apresentadas para comparação)

\begin{tabular}{c|c|c|c|c|c|c}
\hline AMOSTRA & $\mathbf{S i O}_{2}$ & $\mathbf{A l}_{2} \mathbf{O}_{3}$ & $\mathbf{N a}_{2} \mathbf{O}$ & OUTROS & P.F. & RAZÃO Si/AI \\
\hline Caulim - 3,0M & 35,92 & 30,00 & 18,85 & 0,93 & 14,3 & 1,01 \\
\hline Caulim - 5,0M & 35,85 & 29,88 & 18,90 & 0,97 & 14,4 & 1,02 \\
\hline Ganga - 5,0M & 27,19 & 26,48 & 14,75 & 18,98 & 12,6 & 0,87 \\
\hline Sodalita Básica* Teórica & 37,19 & 31,61 & 25,62 & - & 5,58 & 1,00 \\
\hline Sodalita Básica** Teórica & 35,80 & 30,47 & 24,70 & - & 8,96 & 0,99 \\
\hline * Sodadrosodalita Teórica & 36,14 & 30,72 & 18,67 & - & 14,45 & 0,99 \\
\hline
\end{tabular}

\subsection{Influência da concentração molar e do tempo de reação}

Com base nos resultados obtidos, pode-se assumir que nas condições estudadas, sodalitas de duas séries são formadas (sodalita básica e hidrosodalita).

Em presença da solução fortemente alcalina há uma nucleação e precipitação inicial da sodalita básica. Ao passo que o $\mathrm{NaOH}$ é consumido pela conversão da caulinita em sodalita básica, a hidrosodalita é formada pela troca de moléculas de $\mathrm{NaOH}$ e $\mathrm{H}_{2} \mathrm{O}$ presentes na sodalita inicialmente formada e o meio reacional, respectivamente, resultando na fase predominante nas amostras produzidas em tempo e concentrações intermediárias (180 min; 3,0 à 4,5 M, respectivamente) [22, 23]. Vale ressaltar que a composição química da hidrosodalita contém duas moléculas de $\mathrm{NaOH}$ a menos que a sodalita básica. Assim, um possível favorecimento desta fase em detrimento a sodalita básica acarretaria uma economia de 25 \% no consumo de $\mathrm{NaOH}$, o que seria desejável em um contexto do processo Bayer.

Observa-se que durante toda a reação em tempos e concentrações menores não é possível alcançar um equilíbrio, e assim, há uma constante alternância da fase predominante. Em tempos e concentrações maiores um equilíbrio é alcançado, de modo que não se observa mais picos de difração separados, e sim um único pico intermediário.

\section{CONCLUSÕES}

De acordo com os resultados obtidos neste trabalho, dados a partir de um caulim essencialmente puro e de boa cristalinidade, pode-se concluir que:

- A destruição/conversão completa da caulinita em sodalita só ocorreu em tempos de reação superiores a 120 min. A concentração de $\mathrm{NaOH}$ influi positivamente na velocidade de transformação e também no tipo de fase sodalita formada;

- Sodalitas com diferenças na composição química e estrutural foram formadas. Pela comparação dos resultados obtidos por DRX, ATD/TG e EIV pode-se observar que estas fases correspondem aos membros extremos das séries: sodalita básica $\left(\mathrm{Na}_{8}\left[\mathrm{AlSiO}_{4}\right]_{6}[\mathrm{OH}]_{2} \cdot 4 \mathrm{H}_{2} \mathrm{O}\right)$ e hidrosodalita $\left(\mathrm{Na}_{6}\left[\mathrm{AlSiO}_{4}\right]_{6} \cdot 8 \mathrm{H}_{2} \mathrm{O}\right)$;

- A sodalita básica tetrahidratada é primeira a se cristalizar, e se mostrou mais estável, sendo observada em todas as condições reacionais adotadas neste trabalho;

- A hidrosodalita é uma fase secundária formada em condições de tempo e concentração intermediários. Sua formação acontece pela troca de moléculas de $\mathrm{NaOH}$ da sodalita básica formada inicialmente e $\mathrm{H}_{2} \mathrm{O}$ presente na solução lixiviante usada. Dessa forma há uma diminuição de $25 \%$ do $\mathrm{NaOH}$ consumido no processo; e

- Pensando no contexto do processo Bayer, o favorecimento da cristalização da hidrosodalita seria economicamente mais desejável, pelo menor consumo de $\mathrm{NaOH}$.

A partir dos resultados das gangas cauliníticas se observou que o favorecimento da hidrosodalita depende de:

- Maior concentração de caulinita (maior teor de sílica reativa na bauxita processada); 
- Maior tempo de reação; e

- Maior concentração alcalina da solução lixiviante.

Esses dados mostram que o favorecimento da hidrosodalita em detrimento a sodalita básica seria uma possível solução para as bauxitas com alta sílica reativa de Paragominas. No entanto, maiores tempos e concentrações alcalinas despenderiam maiores gastos de energia. Assim, fazem-se necessários estudos posteriores que apontem modificações viáveis do processo Bayer para o favorecimento desta fase.

\section{AGRADECIMENTOS}

A CAPES pelo auxílio financeiro, bolsa de mestrado do primeiro autor. Ao CNPq Edital MCT/CTMineral/VALE/CNPq ${ }^{\circ}$ 12/2009, 550.297/2010-3); e a FAPESPA Edital 01/2010, ICAAF $n^{\circ}$ 027/2011, também pelo apoio financeiro.

\section{BIBLIOGRAFIA}

[1] DNPM, SUMÁRIO MINERAL 2015, http://www.dnpm.gov.br/dnpm/sumarios/sumario-mineral-2015, acessado em Julho 2016.

[2] CARVALHO, A., Bauxitas no Brasil: síntese de um programa de pesquisa, Tese de D.Sc., Universidade de São Paulo, São Paulo, 1990.

[3] HILDEBRANDO, E. A., ANGÉLICA, R. S., NEVES, R. F., et al., "Síntese de zeólita do tipo faujasita a partir de um rejeito de caulim”, Cerâmica, v. 58, pp, 453-458, 2012.

[4] SILVA, H. M., PICANÇO, E., MAURITY, C., et al., "Geology, Mining Operation and Scheduling of the Paragominas Bauxite Mine”, In: Proceeding of the $8^{\circ}$ International Alumina Quality Workshop, pp. 11-16, Darwin, 2008.

[5] KWITKO-RIBEIRO, R., FERREIRA, E. E., AVELAR, A. N., et al., “Automated mineralogy applied to bauxite characterization: a case study”, In: ICSOBA, pp. 86-93, Montréal, 2006.

[6] PAZ, S. P. A., ANGÉLICA, R. S., SCHELLER, T., "X-ray diffraction (XRD) studies of kaolinites to support mineralogical quantification of high silica bauxites from the Brazilian Amazon region”, In: ICSOBA, BX 16-T, 7 pp., Belém, 2012.

[7] PAZ, S. P. A., ANGÉLICA, R. S., NEVES, R. F., “Síntese hidrotermal de sodalita básica a partir de um rejeito de caulim termicamente ativado”, Química Nova, v. 33, n. 3, pp. 579-583, 2009.

[8] MAIA, A. A. B., SALDANHA, E., ANGÉLICA, R. S., et al., "Utilização de caulim da Amazônia na síntese de zeólita A”, Cerâmica, v. 53, pp. 319-324, 2007.

[9] MAIA, A. A. B., ANGÉLICA, R. S., NEVES, R. F., "Estabilidade térmica da zeólita A sintetizada a partir de um caulim da Amazônia”, Cerâmica, v. 54, pp. 345-350, 2008.

[10] SMITH, P., “The processing of high silica bauxites - Review of existing and potential processes”, $\mathrm{Hy}$ drometallurgy, v. 98, pp. 162-176, 2009.

[11] ARMSTRONG, J.A., DANN, S.E., "Investigation of zeolite scales formed in the Bayer process", Microporous and Mesoporous Materials, v. 41, pp. 89-94, 2000.

[12] NASCIMENTO, L.G., PAZ, S.P.A., ANGÉLICA, R.S., et al., "Síntese de zeólitas a partir do rejeito gibbsítico/caulinítico do beneficiamento de bauxita de Paragominas-PA”, In: $57^{\circ}$ Congresso Brasileiro de Cerâmica e $5^{\circ}$ Congresso Iberoamericano de Cerâmica, pp. 1019-1029, Natal, May, 2013.

[13] MELO, C. C. A., PAZ, S.P.A., ANGÉLICA, R.S., et al., "Investigação da formação de sodalita Bayer no processo de refinamento de alumina”, In: $57^{\circ}$ Congresso Brasileiro de Cerâmica e $5^{\circ}$ Congresso Iberoamericano de Cerâmica, pp. 4199-4210, Natal, May, 2013.

[14] SILVA, L. N., PAZ, S. P. A., ANGÉLICA, R. S., et al., "Estudo cinético de formação de sodalita a partir de um rejeito de caulim do rio Jari - PA", 55 Congresso Brasileiro de Cerâmica, pp, 601-612, Porto de Galinhas, May, 2011.

[15] GUALTIERI, A., NORBY, P., ARTIOLI, G., et al., "Kinetic study of hydroxysodalite formation from natural kaolinites by time-resolved synchrotron powder diffraction”, Microporous Materials, v. 9, pp. 189201, 1997.

[16] PLANÇON, A., GIESE, R. F., SNYDER, R., “The Hinkley index for kaolinites”, Clay Minerals, v. 23, pp. 249-260, 1988.

[17] BARNES, M. C., ADDAI-MENSAH, J., GERSON, A. R., “The kinetics of desilication of synthetic 
spent Bayer liquor seeded with cancrinite and cancrinite/sodalite mixed-phase crystals”, Journal of Crystal Growth, v. 200, pp. 251-264, 1999.

[18] BARNES, M. C., ADDAI-MENSAH, J., GERSON, A. R., "The kinetics of desilication of synthetic spent Bayer liquor and sodalite crystal growth”, Colloids and Surfaces, v. 147, pp. 283-295, 1999.

[19] HELLER-KALLAI, L., LAPIDES, I., "Reactions of kaolinites and metakaolinites with $\mathrm{NaOH}$ - comparison of different samples (Part 1)”, Applied Clay Science, v. 35, pp. 99-107, 2007.

[20] GOUGAZEH, M., BUHL, J.-Ch., "Synthesis and characterization of zeolite A by hydrothermal transformation of natural Jordanian kaolin", Journal of the Association of Arab Universities for Basic and Applied Science, v. 15, pp. 35-42, 2014.

[21] FELSHE, J., LUGER, S., BAERLOCHER, CH., “ Crystal structures of the hydro-sodalite $\mathrm{Na}_{6}\left[\mathrm{AISiO}_{4}\right]_{6} 8 \mathrm{H}_{2} \mathrm{O}$ and of the anhydrous sodalite $\mathrm{Na}_{6}\left[\mathrm{AISiO}_{4}\right]_{6}$ ", Zeolites, v. 6, pp. 367-372, Sep, 1986.

[22] FELSHE, J., LUGER, S., "Phases and thermal decomposition characteristics of hydro-sodalites $\mathrm{Na}_{6+\times}\left[\mathrm{AISiO}_{4}\right]_{6}(\mathrm{OH})_{\times} \times n \mathrm{H}_{2} \mathrm{O}$ ”, Thermochimica Acta, v. 118, pp. 35-55, 1987.

[23] MOLOY, E. C., LIU, Q., NAVROTSKY, A., "Formation and hydration enthalpies of the hydrosodalite family of materials”, Microporous and Mesoporous Materials, v. 88, pp. 283-292, 2006.

[24] KHAJAVI, S., SARTIPI, S., GASCON, J., JANSEN, J. C., KAPTEIJN, F., "Thermostability of hydroxy sodalite in view of membrane applications", Microporous and Mesoporous Materials, v. 132, pp. 510-517, 2010.

[25] ENGELHARDT, G., FELSCHE, J., SIEGER, P., “ The hydrosodalite $\mathrm{Na}_{6+x}\left[\mathrm{SiAlO}_{4}\right]_{6}(\mathrm{OH})_{\mathrm{x}} \mathrm{nH}_{2} \mathrm{O}$ : formation, phase composition, and de- and rehydration studied by ${ }^{1} \mathrm{H},{ }^{23} \mathrm{Na}$, and ${ }^{29} \mathrm{Si}$ MAS-NMR spectroscopy in tandem with thermal analysis, X-ray diffraction, and IR spectroscopy”, Journal of the American Chemical Society, v. 114, pp. 1173-1182, 1992.

[26] NASKAR, M. K., KUNDU, D., CHATTERJEE, M., “ Effect of process parameters on surfactant-based synthesis of hydroxy sodalite particles”, Materials Letters, v. 65, pp. 436-438, 2011.

[27] RABO J. A., Zeolite Chemistry and Catalysis, American Chemical Society, Washington, 1976. 